\title{
Underlying histopathology of peripheral nerve injury and the classical nerve repair techniques
}

\author{
1 Plastic Surgery Department, "Bagdasar Arseni" Emergency \\ Hospital, Bucharest, ROMANIA \\ 2 Plastic Surgery Department, "Grigore Alexandrescu" Emergency \\ Pediatric Hospital, Bucharest, ROMANIA
}

Marin Andrei ${ }^{1}$, Mihai Ruxandra loana ${ }^{1}$, Enescu Dan Mircea ${ }^{2}$

\begin{abstract}
A much-debated subject in the last 20 years, the recovery after peripheral nerve injury still remains one of the most researched themes of our days. Although the central nervous system has not exhibited any ground-breaking discoveries in matters of healing through surgical procedures, this is not the case for the peripheral nervous system (PNS). The PNS recovery after injury has improved over the years so we now speak of time and percentage of rehabilitation. The increased interest for this subject is a result in the development of the medical technique, that allowed the creation of new molecules capable to improve the regeneration rate. Furthermore, the evolution in diagnostic parameters, as well as the possibility of a thorough follow-up, contributed to the ascending research of this field. One must not forget that all experimental studies have as endpoint obtaining safe and reproducible solutions which can be applied in treating patients with peripheral nerve injury. We will briefly present the microscopic events that occur following a peripheral nerve injury, the key factors which influence their regeneration as well as the classical techniques used to repair them. However, the most intriguing topic in nerve regeneration is not related to the surgical procedure (considered to be the Gold Standard in whole nerve injury), but rather the helping substances that facilitate a faster and better recovery.
\end{abstract}

\section{INTRODUCTION}

The first world war was the trigger that put peripheral nerve injuries under the microscope.[1] The lack of motility/sensitivity generated a major distress that affected the quality of life of war veterans. This resulted in a greater study of the anatomy, physiology and histopathology of peripheral nerves, Platt describing a series of 26 patients who underwent surgery for nerve repair.[2]

Several authors came up with a series of classification, taking into account the degree of injury and the histologic aspects, the most known being the Seddon classification (3 degrees of injury - neuropraxia, axonotmesis, neurotmesis) [2] and the Sunderland classification (5 degrees, splitting the axonotmesis degree into 3 different categories).

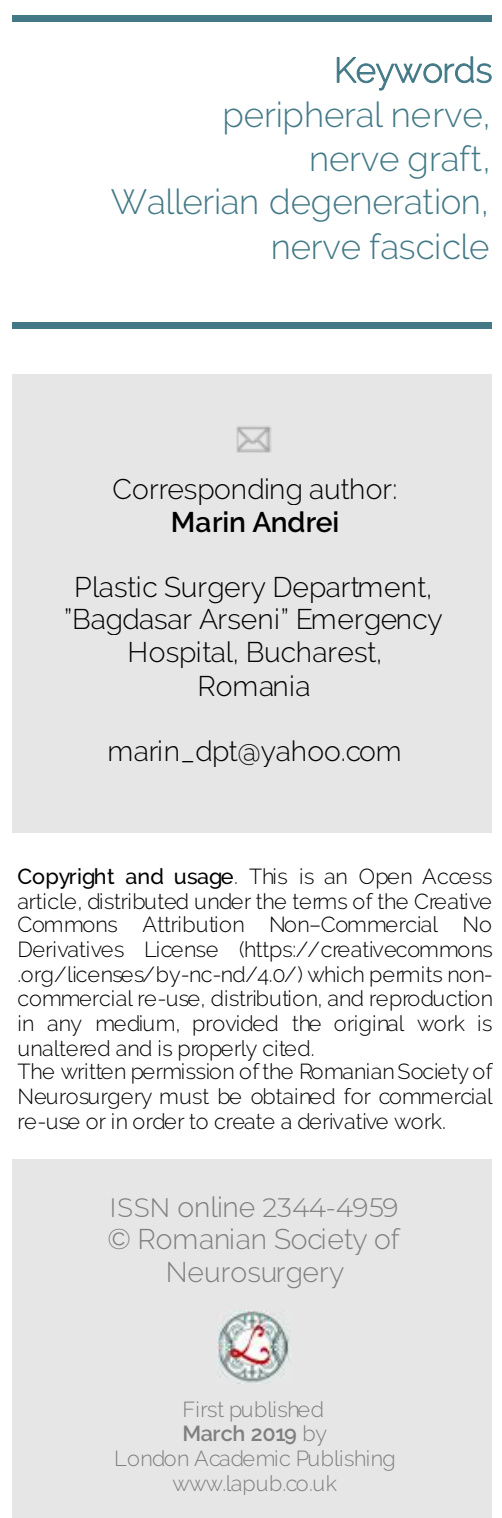


[4] Mackinnon introduces a 6th type of nervous injury - a combination of different degrees of nerve injuries.[5]

Neuropraxia represents a block in the conduction of the nerve impulse without any histologic lesions. Its recovery is self-limited in 6-12 weeks. The second degree injury (axonotmesis) involves the interruption of the axon keeping the integrity of the neural tube. This leads to anterograde Wallerian degeneration as well as a short retrograde nerve degeneration. The intact endoneural tube consisting of epinerve, perinerve, endonerve and the sheath of Schwann cells ensures the premises of a good recovery.

Sunderland's 3rd degree lesion associates an endonerve injury, which implies a minimum fibrosis; for this reason, the recovery will be slightly deficitary. 4th degree lesion implies keeping only the epinerve intact, a surgical intervention being absolutely necessary. Neurotmesis (5th degree lesion) consists in the complete interruption of the nerve. With the exception of the 1st degree injury, all others will suffer processes of degeneration followed by regeneration.

\section{OVERVIEW ON EVOLUTION AND COMPLICATIONS IN NERVE INJURIES}

Nervous degeneration. The disruption of the connection between axon and neural body triggers the initiation of axonal disintegration distal to the level of injury. This is called Wallerian degeneration and it is an autolytic process of cleansing the distal stump. The axoplasma and the myelin sheath will be disintegrated, followed by a phagocytic process performed by macrophages and Schwann cells. The Wallerian degeneration takes place strictly in the myelinated fibers of the distal nerve. The Schwann cells will proliferate, changing their fenotype and reducing the synthesis of myelin. The cells will then align to form the Bugner bands (endoneural tubular structures capable of directing nerve fibers). Their role is to guide the neural sprouts from the proximal end on the right path towards the original endorgan. This guidance occurs under certain neurotrophic factors, as well as neurotropic agents such as: NGF - nerve growth factor, glial maturation factor-b, GFAP - glial fibrillary acidic protein, NCAM cellular adhesion molecules, GGF - glial growth factor, BDNF - brain derived neurotrophic factor, IGF-1 insulin growth factor.[6]

There are also a series of agents which modulate the inflammatory process or intervene in the cellular change: cytokines - IL1, IFNy, IL6 si IL12, TNF-a, the latter being responsible for macrophage recruitment.

Aside from the Wallerian degeneration, there is also a certain degree of retrograde degeneration in the proximal segment. The axon proximal to the injury depends on the state of the cellular body - it either survives and regenerates or it dies. The last surviving Ranvier node modifies his structure and biochemistry, preparing for axonal regeneration.

Nervous regeneration. There is a de novo restoration of the axon through a process called axonal sprouting. This is realized through the synthesis of the cellular components and the renewal of the original axonal architecture. At the proximal site the neural sprouts advance distally and form together with the axon a regenerative unit. The distal area of each axonal sprout presents a tumescence called growth cone, which contributes to guiding the nervous regeneration. The axonal sprouts cross the lesion area, reaching the distal end. Once in contact with a Bugner band, the axonal sprouts will be covered by the cytoplasm of the Schwann cells and advance through the endoneural tubes to reach the target organs.[7] In case of a misalignment, these will advance towards other organs, different from the one they innervated initially. If these sprouts don't advance correctly, they may end their elongation process by forming a neuroma.

The advancement of the growth cone is done through contact guiding (the local environment playing an important role), as well as neurotropic factors. Tissue specificity implies preferential growth of the axonal sprouts towards nervous tissue structure, and inhibiting the paths towards other structures (muscle or tendon). The nervous fibers also posses the capacity to differentiate between motor and sensitive nerves in mixt nervous trunks. Topographic specificity implies correct reinnervation of the right muscle (motor fibers) or cutaneous area (sensitive fibers). End organ specificity refers to re-innervation of the correct sensitive organ (Paccini, Krause, Ruffini) or muscle fiber (fast or slow). 


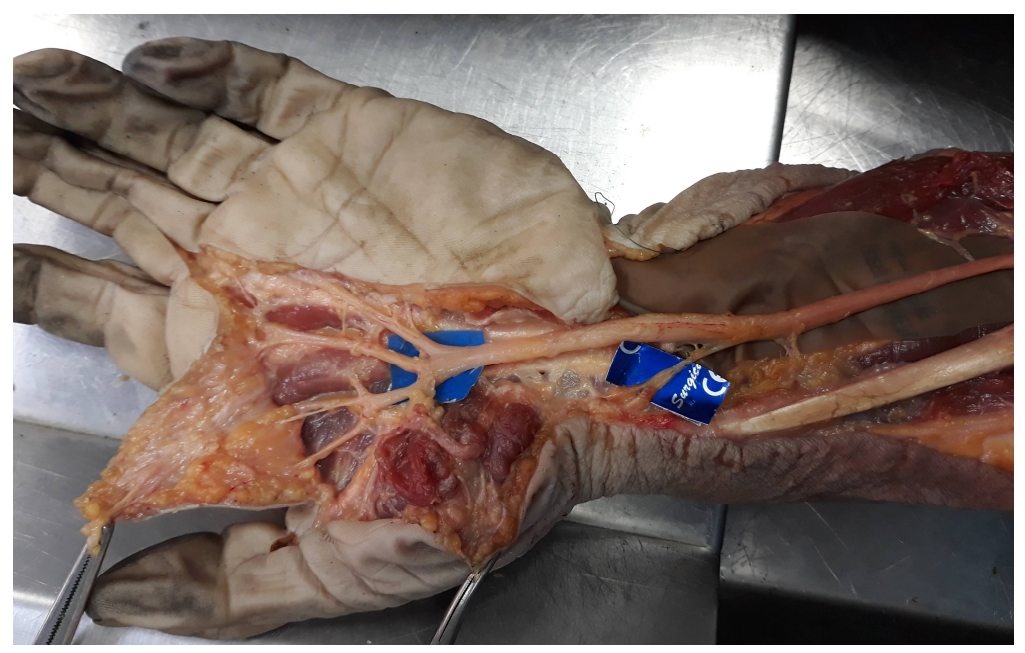

CADAVER DISSECTIONS - median nerve in forearm and hand (above) and ulnar nerve at elbow region (right).

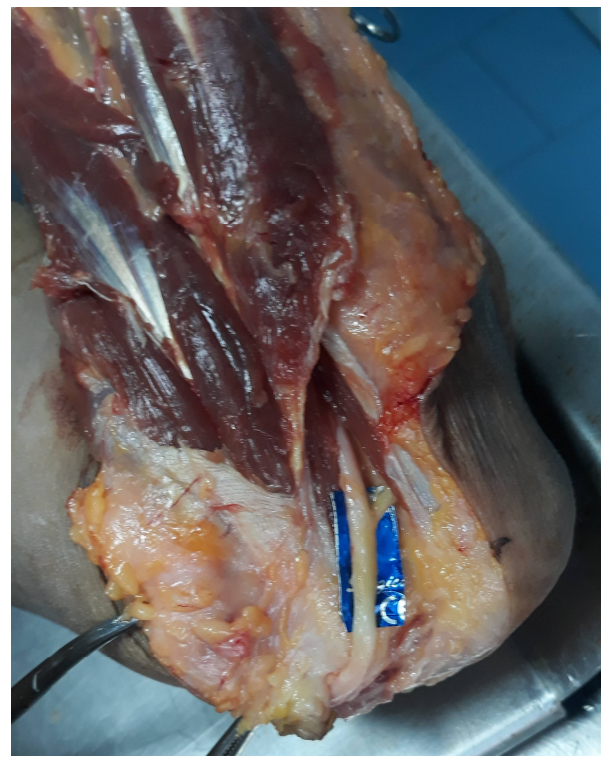

\section{Factors which influence the regeneration process} The division of the axon determines complex processes which aim the nervous restoration. The surgical repair creates favorable conditions for nervous re-innervation. The stages for nerve repair are: debridement of the injured area, approximation and nerve suture.

The factors that influence the final outcome are: factors relating to the patient (age, habits such as smoking), timing for nerve repair, the degree of injury, injury mechanism, level of injury, type of nervous repair, surgical technique (atraumatic, following the tension free principle), as well as the experience of the surgeon and the availability of proper microsurgery instruments as well as a microscope.

Young patients have a much better capacity of recovery compared to older patients. In model rat experiments, it has been proven that smoking slows down the regeneration of peripheral nerves. Other medical conditions such as diabetes, hypothyroidism or peripheral vascular diseases interfere with the nervous regeneration.

The timing is an important issue in nerve repair. Primary repair is done in the first 72 hours. Between 72 hours and 7 days, there is a delayed primary repair and after this time - secondary repair. The sharp object injuries with minimal contamination benefit from primary repair with favorable results. For crush injuries or avulsions where viable and nonviable tissue is more difficult to set apart - a waitand-watch approach is the appropriate therapy. In case no improvement is seen 3 months from injury, a surgical intervention to prevent denervation and muscle loss is recommended.

The degrees of injury were previously presented. 1st and 2nd degree injuries have more favorable results compared to the higher degrees of injury. The mechanism of injury is a predictive factor - cutting injuries have better results than crush injuries. The level of injury constitutes another factor - proximal lesions have poorer results compared to distal ones. The explanation is that it takes a longer time for the axon to reach its end organ when the lesion is proximal; furthermore, the more proximal the injury, the more nervous fibers that the nerve contains (sometimes both motor and sensitive), which requires a perfect alignment so that each fiber finds the right way.

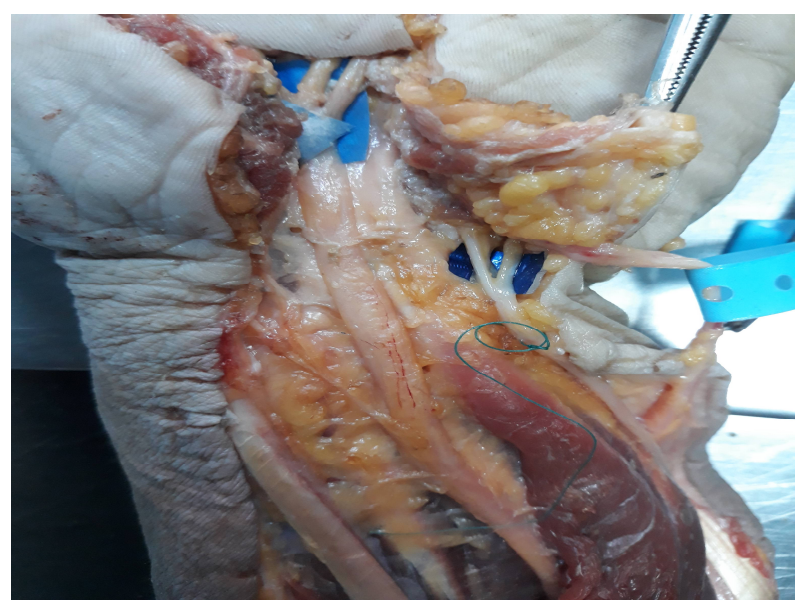

Cadaver dissection: median and ulnar nerve at the wrist region (representation of the ulnar nerve bifurcation at this site). 


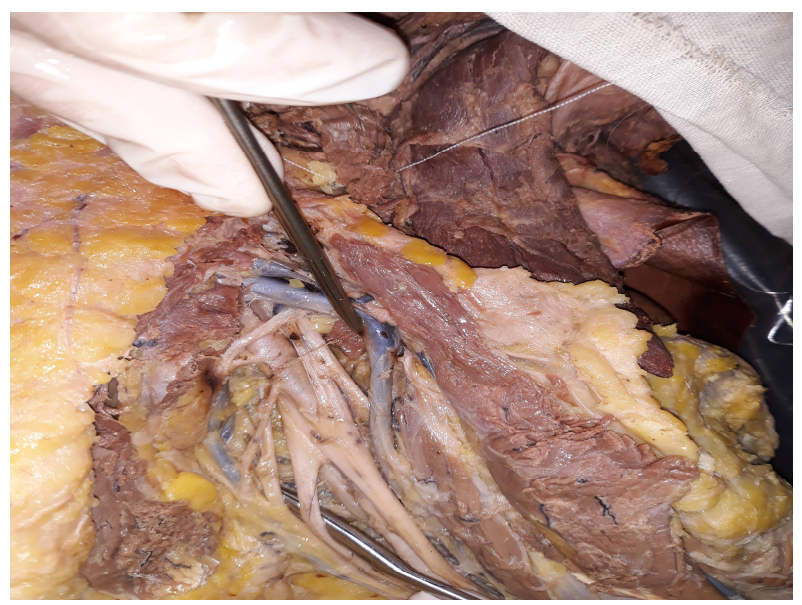

Cadaver dissection: median and ulnar nerve at the wrist region (representation of the ulnar nerve bifurcation at this site);

\section{Classic repair techniques}

There are 3 classic nervous sutures: epineural, perineural and epiperineural.

The epineural technique requires nervous endings isolation, placing a contrasting background behind them, excision of the injured tissues. The nervous endings are trimmed for a suitable match and are then arranged so that the inner fascicles correspond to one another. The sutures are place at 1800 and the rest are placed at half the distance. Main disadvantage is that the nerve is connected only on the outside, the fascicles being able to slide and follow wrong regeneration paths. On the other hand, this suture has the advantage of a minimal fibrosis.

Repairing groups of fascicles is a more precise technique in terms of alignment but it has the disadvantage of transferring the tension from the epinerve directly to the fascicles. It also requires more sutures for a well distributed tension, which implies more fibrosis.
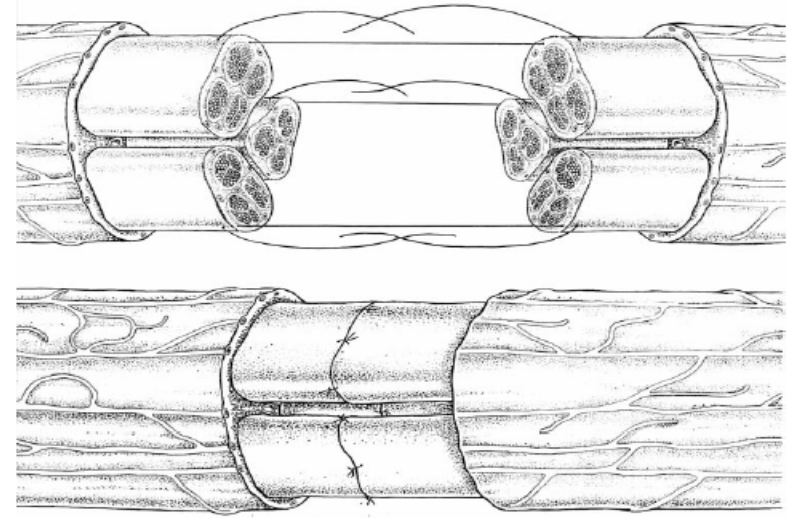

GROUP FASCICLE REPAIR

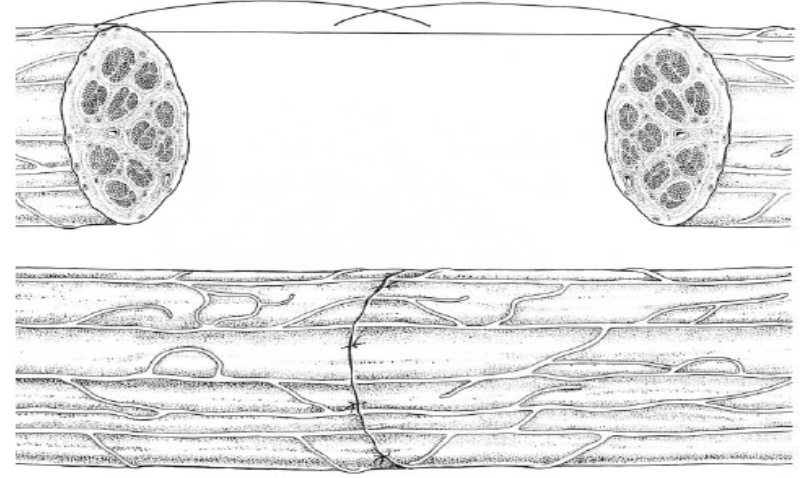

EPINEURAL REPAIR

The fascicular repair is done by suturing each fascicle individually and may be indicated in mixt nerves where there is a high probability of unsuitable axonal growth (sensitive nerves growing in the motor nerves' path). In the case of fascicular suture, the internal epinerve is dissected and the fascicles are sectioned at different sites and repaired by 1-2 sutures. Despite an almost perfect alignment, there is a great degree of fibrosis so the outcome doesn't bring better results.

\section{Nerve grafting}

When the nervous defect exceeds the length of a direct suture, the gap must be filled by a structure that can guide the nervous regeneration. The nerve graft is the Gold Standard in this case. It acts as a path that the new axon passes, having the trophic support of the Schwann cells.

There are several types of grafts: autografts (from the same person), isografts (from identical twin), allografts (from the same species) and xenografts (from different species). When talking about allografts and xenografts, an important issue is the immune reaction of the organism and the immunosuppression used to prevent graft vs host reaction.

Allografts can be obtained from cutaneous nerves or nerve trunks. The harvest doesn't determine a high degree of functio laesa in case of cutaneous nerves but nerve trunks are harvested only in the case of a fully compromised muscle (complete muscle denervation). Graft diameter is of great importance in a successful transfer because in grafts with a diameter above $3 \mathrm{~mm}$ there is a great risk of ischemia with possible central necrosis.

The shortcomings in using an autologous graft is that it requires a donor site sacrifice. Furthermore, 
all grafts will suffer the same changes as in the distal end of an injured nerve and the axon will be forced to pass through 2 different sutures. Sometimes, in the distal suture, the fibrosis can be so great it can hinder the axon from running his course. This is the case for long grafts and this can be prevented by sectioning distal end of the graft in a second intervention. Some authors recommend only the proximal end of the graft to be sutured in a first stage and the distal in a second stage, once the neurotisation is complete. If the block exists, resection and resuture is mandatory.

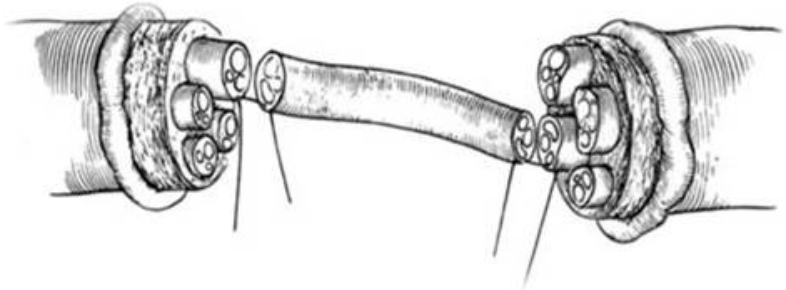

Another important issue is the difference in calibre. There are 5 types of fascicle distribution which should be handled differently. The single fascicle arrangement (the nerve containing one big fascicle) or the arrangement with 2-4 big fascicles - the graft should cover the whole cross section of the injured nerve. In the case with 5-12 fascicles nerves, these contain a higher amount of connective tissue. Therefore, the suture is best done for each fascicle individually.

The polifascicle nerves contain a high level of connective tissue which should be excised to prevent graft adherence to connective tissue. Single suture similar to the previous type is the proper nerve repair in this case. The last type is the polifascicle arrangement in which the fascicles are randomly distributed and has a high risk of misalignment. It doesn't allow a fascicle group identification therefore the interfascicle dissection is futile. Grafts similar in diameter and sectional topography are sutured to this type of nerve. If the nerve diameter exceeds the one of the graft, a longitudinal dissection of the graft is performed and only a part of the graft is used.

There are several types of nerve grafts: free transferred, pedicled, cable grafts, trunk grafts, fascicle and interfascicle grafts.

Free transferred grafts don't contain any vascular source, the inosculation process taking place 3-4 days after the nerve suture. These first days, the nerve is vascularized through diffusion. The large caliber grafts may suffer from ischemia, existing a risk of central necrosis.

The pedicle graft is used when there are simultaneous injuries to 2 nerves in proximity one to another (median and ulnar nerve). The proximal ends of the 2 nerves are sutured, while the ulnar nerve is sectioned as proximal as possible. The ulnar nerve however isn't deprived of it's vascular supply. After neurotization, the ulnar nerve graft is transposed inferiorly to restore the trajectory of the median nerve. At this time, the ulnar graft is sutured at the distal end of the median nerve.

The free vascularized nerve graft represents the transfer of the nervous tissue alongside his vascular supply. This technique can be used when the receiving site is not favourable for regeneration (high degree of fibrosis). This operation is reserved for extremities or fingers because the intervention can be quite laborious.

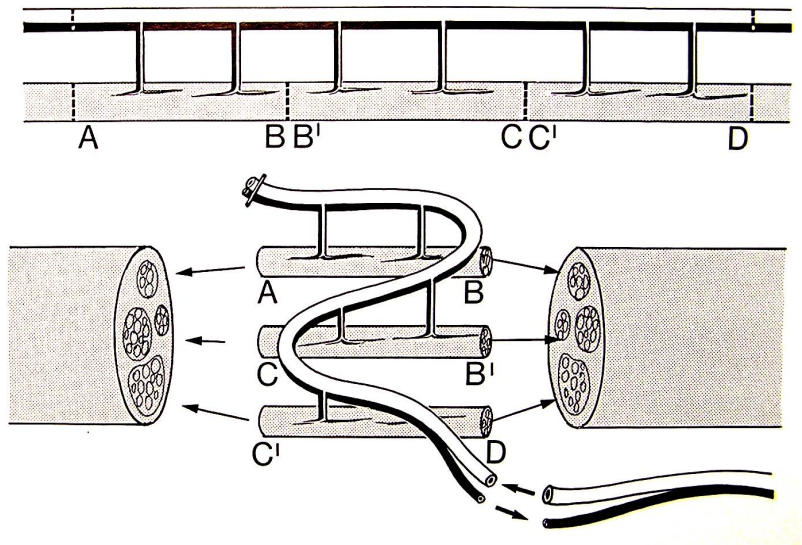

The interfascicle graft is used for groups of fascicle cooptation. It implies excision of the external epinerve at the proximal end, dissection on fascicle groups, each group being individualized at different distances to increase suture resistance. Same technique is used in the distal end of the graft. The individual fascicle graft is used for the repair of terminal nerves (digital nerves or the ulnar nerve at the elbow region which contains a small number of fibres.

The cable nerve graft is formed by harvesting several cutaneous small nerves, which are placed parallel to cover a surface equal to the diameter of the injured nerve. This was replaced by the interfascicle nerve graft because the cable nerve grafts involve putting larger amounts of connective tissue in contact with nervous tissue, thus inhibiting the nervous regeneration. The trunk graft is no 
longer in use because of its large diameter that caused multiple central necrosis.

\section{CONCLUSIONS}

This review aims to present the most important aspects of peripheral nerve regeneration, being a foundation stone for a very complex subject. Having described the underlying processes involved in peripheral nerve degeneration, as well as the classical repair techniques, it is obvious that progress in this field has been done. However, the technological progress as well as the development of new molecules that can aid nerve regeneration show that there is always room for improvement in the future.

\section{REFERENCES}

1. Bristow WR (1947) Injuries of peripheral nerves in two world wars. BrJ Surg 34(136):333-348

2. Platt, H.: On the Results of Bridging Gaps in Injured Nerve Trunks be Autogenous Fascial Tubulization and Autogenous Nerve Grafts. The British Journal of Surgery 7:384-9, 1919-1920

3. Kaya, Y. \& Sarikcioglu, L.: Sir Herbert Seddon (1903-1977) and his classification scheme for peripheral nerve injury, Childs Nerv Syst (2015) 31: 177. https://doi.org/10.1007/s00381-014-2560-y

4. Sunderland S (1951) A classification of peripheral nerve injuries producing loss of function. Brain 74(4):491-516

5. Chhabra, A., Ahlawat, S., Belzberg, A., \& Andreseik, G. (2014). Peripheral nerve injury grading simplified on MR neurography: As referenced to Seddon and Sunderland classifications. The Indian journal of radiology \& imaging, 24(3), 217-24.

6. Kronenberg, J., Merkel, L., Heckers, S. et al. J Mol Neurosci (2019). https://doi.org/10.1007/s12031-019-1258-8

7. Caillaud $M$, Richard L, Vallat JM, Desmoulière $A$, Billet $F$. Peripheral nerve regeneration and intraneural revascularization. Neural Regen Res. 2019;14(1):24-33. 